\title{
DEVELOPMENT AND CHARACTERISATION OF A HEAD CALIBRATION PHANTOM FOR IN VIVO MEASUREMENTS OF ACTINIDES
}

${ }^{1}$ G. Gualdrini, ${ }^{\mathbf{1}_{\mathrm{P}} \text {. Battisti, }}{ }^{\mathbf{2}}$ R. Biagini, ${ }^{\mathbf{2}} \mathrm{P}$. De Felice, ${ }^{\mathbf{2}}$ A. Fazio, ${ }^{\mathbf{3}} \mathrm{P}$. Ferrari

1; Ente Nazionale per le Nuove Tecnologie, l'Energia e l'Ambiente - Istituto per la Radioprotezione v. Dei Colli 16 I-40136 Bologna (Italy)

2; Ente Nazionale per le Nuove Tecnologie, l'Energia e l'Ambiente - Istituto Nazionale di Metrologia delle Radiazioni Ionizzanti v. Anguillarese, 301, I-00060 S. M. Galeria-Roma (Italy)

3; Degree in Physics, Physics Department-University of Bologna Viale C. Berti Pichat 6/2 I-40127 Bologna (Italy)

Abstract: The investigation of actinides internal contamination in human body makes use of a variety of techniques. In large scale screening procedures the "in vivo" measurement of bone ${ }^{241} \mathrm{Am}$ burden via the determination of the nuclide activity in the skull is often used. To this purpose adequate calibration procedures and standard phantoms are needed.

The present paper summarises the studies and technical procedures followed for the development of a calibration phantom based on a commercial Alderson angiographic head in which a set of 24 ${ }^{241}$ Am point sources were imbedded.

A theoretical study was first carried out, at the ENEA Institute for Radiation Protection, using the MCNP4-B Monte Carlo code to determine the point source distribution that closer approximate a homogeneous bone contamination. The numerical models were also used to evaluate the resulting degree of approximation.

The point sources were prepared at the ENEA National Metrology Institute for ionising radiation quantities and are traceable to the Italian national standard of radionuclide activity. The sources were prepared quantitatively dispensing a liquid solution onto a plastic film. The activity of each source was checked by gamma-ray spectrometry and the reproducibility of the activity values was determined. Each source was then placed in the optimum position, given by the Monte Carlo modelling, by a precision mechanical device. The phantom was finally used to calibrate a counting system operating at the ENEA Institute for Radiation Protection. 
The paper reports the main theoretical and experimental aspects of this work also discussing the results of the first calibrations.

\section{1-INTRODUCTION}

The evaluation of ${ }^{241} \mathrm{Am}$ bone burden through "in vivo" measurement of the nuclide activity in the skull is one of the most used techniques in large scale screening of actinides previous internal contamination $^{[1]}$.

Taking into account that, for radiation protection purposes, very high sensitivity, accuracy and precision are requested in this kind of measurements, the major problem is often due to the difficulty to develop suitable standards for the calibration of the detection system.

The present paper summarises the studies and the technical procedure followed for the development and characterisation of a head calibration phantom ${ }^{[2]}$ for "in vivo" measurement of ${ }^{241} \mathrm{Am}$ based on a commercial one, normally employed for angiographic analysis calibration. The main purpose was to develop a phantom in which a limited number of point sources should well approximate the homogeneous distribution typical of an Americium internal contamination ${ }^{[3]}$.

\section{2- PREPARATION OF THE HEAD PHANTOM}

A selection of commercial head phantoms was made, in order to find the one with the best anthropomorphic characteristics. The Alderson ${ }^{\mathrm{TM}}$ phantom for angiographic analysis was chosen (figure 1). Its constituents (as kindly supplied by the manufacturer ${ }^{[4]}$ ) are:

- Alderson ${ }^{\mathrm{TM}}$ cortical bone $\left[\rho=1.88\left(\mathrm{~g} / \mathrm{cm}^{3}\right) ; 60 \mathrm{keV} \mu=0.587\left(\mathrm{~cm}^{-1}\right)\right]$

- Alderson $^{\mathrm{TM}}$ trabecular bone $\left[\rho=1.17\left(\mathrm{~g} / \mathrm{cm}^{3}\right) ; 60 \mathrm{keV} \mu=0.274\left(\mathrm{~cm}^{-1}\right)\right]$

- Alderson $^{\mathrm{TM}}$ soft tissue $\left[\rho=1.09\left(\mathrm{~g} / \mathrm{cm}^{3}\right) ; 60 \mathrm{keV} \mu=0.223\left(\mathrm{~cm}^{-1}\right)\right]$ 


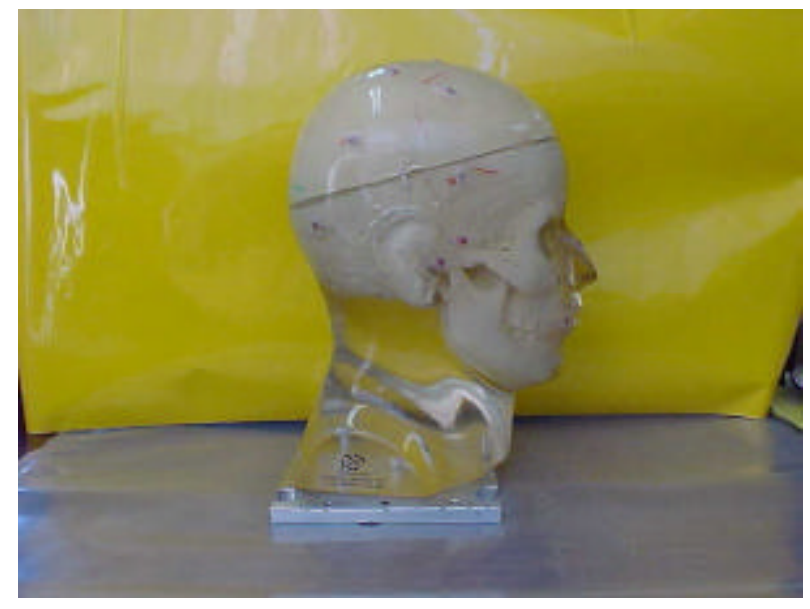

Fig. 1: Lateral view of the Alderson ${ }^{\mathrm{TM}}$ phantom

The following requirements were taken into account in the preparation and characterisation of the radioactive sources: small volume (quasi point-) of radioactive material, homogeneous activity values, precision source positioning inside the head phantom.

The point source geometry was chosen as it minimises the source volume and source selfabsorption. The point sources were prepared depositing $5 \mu \mathrm{L}$ of a liquid ${ }^{241} \mathrm{Am}$ standard solution (0.5M HCl, containing, at 10 March 1999, $25.68 \mathrm{kBq} / \mathrm{g}$ of ${ }^{241} \mathrm{Am}$, with a combined standard uncertainty of $1.5 \%$ ) onto $5 \mathrm{~mm}$ diameter and $1 \mathrm{~mm}$ thick polystyrene disks and evaporating the solution under infrared lamp. The standard solution was calibrated by the ENEA well-type ionisation chamber, and was therefore traceable to the Italian national standard of radionuclide activity. The diameter of the dried radioactive deposit was about $1.5 \mathrm{~mm}$. The active disks were then covered by similar inactive disks with a double adhesive paper in between the two disks.

A first set of standard point sources were prepared by quantitative deposition of the radioactive solution by a polyethylene picnometer. This method allows an accurate determination (combined standard uncertainty of the mass measurements of $0.05 \%$ ) of the deposited solution mass. By this method a number of 35 standard sources were prepared with average deposited solution mass of 5.4 mg and experimental standard deviation of $26 \%$. The source activities ranged from 94.4 to 224.9 Bq. This was not considered satisfactory with respect to the reproducibility needs. Each source was also counted on a gamma-ray spectrometry system, based on a p-type HPGe coaxial detector with 
$15 \%$ relative efficiency, on top of the detector window, for a counting time of $1000 \mathrm{~s}$. The corresponding full-energy peak efficiency values showed an experimental standard deviation of 2.7\%. A comparison of this value with the net peak area standard uncertainty based on counting statistics $(2.2 \%)$, limited other important sources of variability.

A second set of sources was then prepared. In order to obtain more reproducible source activity values, a Pipetman ${ }^{\circledR}$ P20 Gilson pipette was used instead of the plastic picnometer. A constant volume of $5 \mu \mathrm{L}$ was set and polypropylene tips were replaced after each single deposition as droplets frequently remained on the end of the tip. A number of 19 sources were prepared by this second procedure and gamma counted, as described before, in order to get the activity on the base of the average efficiency value previously determined. An average activity of $122.9 \mathrm{~Bq}$ with experimental standard deviation of $2.9 \%$ was obtained. This is $4.3 \%$ lower than the activity value $(128.4 \mathrm{~Bq})$ calculated on the base of the activity concentration of the starting solution and its delivered volume. This discrepancy was only in part explained with the accuracy of the pipette (2\% declared by the constructor). As a conclusion of this analysis it was decided to group the two sets of point sources and to rely the source characterisation on the spectrometric measurements. The frequency distribution of the measured activity values for the $54(35+19)$ sources is reported in figure 2 .



Figure 2: Frequency distribution of the activity values of the 54 standard point sources. The activity values are those obtained from gamma-ray spectrometry measurements. 
The 24 sources needed for the phantom were then selected among all the sources available in the frequency peak between 115 and $135 \mathrm{~Bq}$ (figure 2) with an average activity of $122.4 \mathrm{~Bq}$ and experimental standard deviation of $2.7 \%$. Of the 24 sources selected, 6 were taken from the set obtained by the picnometer method and the remaining from that obtained by the pipette method.

The calibration certificate of the point sources reported the activity value of each source, as obtained by the spectrometric measurement, with its associated uncertainty. The main uncertainty components were (relative standard uncertainty): $0.5 \%$ (dead time), 2.2\% (counting statistics), $1.5 \%$ ( counting efficiency), giving a combined standard uncertainty of $2.7 \%$.

The head phantom was mounted on a spherical co-ordinates rotating base that was positioned onto the working plane of a precision milling machine by which a number of 24 cylindrical holes were made on the head phantom. The head position and the hole depth were chosen to reach the bottom of each hole at the co-ordinates given by the theoretical modelling (described later). As far as possible the drilling direction was set orthogonal to the local head surface. Each source was then mounted on a plastic piston that was inserted into the corresponding hole whose protruding part was finally cut.

\section{3- CHARACTERISATION OF THE HEAD PHANTOM}

The main purpose of the work was to develop a head calibration phantom in which a heterogeneous distribution of 24 nearly identical point sources should approximate the homogeneous distribution that Americium really has in the bone. The two main steps of the procedure were Monte Carlo modelling of the plastic head and optimisation of source placing.

\section{3-1 Monte Carlo modelling of the plastic head}

The MCNP (version 4B) ${ }^{[5]}$ Monte Carlo code was used. This is a powerful 3-dimensional multipurpose code developed at the Los Alamos National Laboratory. It can solve transport problems of 
neutrons, photons and electrons in a fully coupled way (i.e. it is possible to follow the secondary particles created during interactions). A powerful geometric package, based on boolean algebra operators, allows to define 3-D complex geometries using the first, second, fourth (torii) order surfaces that MCNP handles. Effective variance reduction techniques and a detailed statistical package make MCNP a reliable radiation transport code widely renowned for its many applications. The Monte Carlo model of the experimental phantom was the most critical point of the procedure. It implied the knowledge of the bone mass distribution throughout the skull. The problem was in fact to determine 24 nearly equal volume skull zones where to place the sources that had to be representative of the radioactive content of the stated bone volume. A first implemented mathematical model, based on experimental measurements of bone thicknesses and weights of a didactic skull phantom, put in evidence that a more rigorous technique was needed to model the Alderson $^{\mathrm{TM}}$ phantom.

Using a Computed Tomography (Ge Hi Speed Adv RP) a number of 248, $1 \mathrm{~mm}$ thick, phantom axial slices were obtained. Only a subset of them were used for the production of the 3-dimensional MCNP model of the skull, increasing the number of the slices, mathematically treated, when quick geometry variations, along the vertical axis, needed more details.

Each slice of the subset was studied in order to determine which mathematical equations, among those handled by MCNP, could better fit the CT contours. An example of a CT slices together with its MCNP representation is shown in figure 3.

The final geometry is made of 54 layers, 277 MCNP cells and 1929 surfaces. In figure 4 a SABRINA $^{[6]} 3$-dimensional plot of the complete skull is shown. The capability of the MCNP code to evaluate spatial cell volumes was employed to determine the ${ }^{241} \mathrm{Am}$ sources positions. In fact each of the 24 sources should have been placed in the centroid of 24 equal volume sub-regions to obtain a nearly homogeneous distribution of the radioactivity. 



Fig.3: A CT scan of head calibration phantom (left), at cheek height, and its MCNP representation

\section{3-2 Optimisation of source positioning}

The positions chosen in the distribution of point sources strongly influence the counting system efficiency. As a matter of fact the closer to the head surface is a detector, the easier the condition of a homogeneous distribution can fail. For each measurement position a correction factor between practical heterogeneous and theoretical homogeneous configuration was defined through the Monte Carlo modelling of the experiment. 


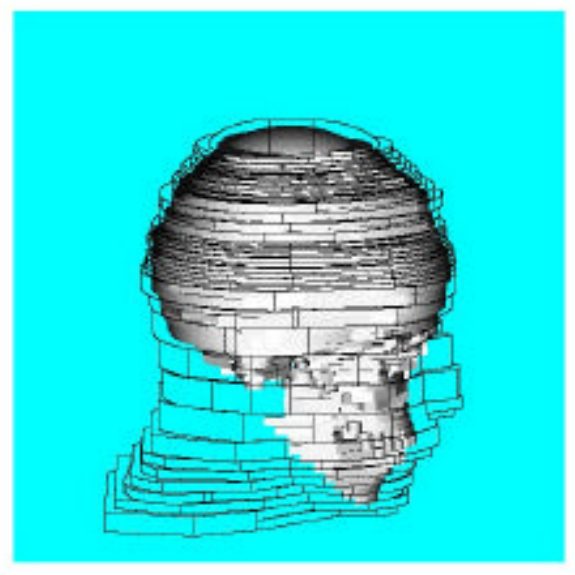

Fig.4: SABRINA plot of the MCNP Alderson ${ }^{\mathrm{TM}}$ phantom with soft tissue transparent

MCNP allowed to obtain an azimuthal-polar mapping of the photon fluence within the detector ROI

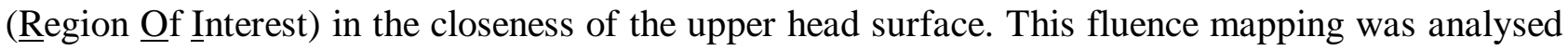
at various distances from the phantom and allowed to:

- Optimise the two Ge detector positions for the calibration;

- Determine the behaviour of the correction factor for the fluence as a function of azimuthal-polar angle and of the distance from the head surface.

The procedure adopted can be briefly summarised as follows:

- A quarter of a hollow sphere was modelled in the top front area of the head (figure 5);

- It is subdivided in $15^{\circ}$ azimuthal and polar angles;

- Each of so defined directions represents a possible scoring area for the detector. This allowed subdividing the response (photon fluence contributing to the total absorption peak) in $13 \times 6+1$ circular areas of the same size of the detector window;

- The same method was applied both for homogeneous source distribution and for the 24 sources distribution;

- The results obtained were graphically compared (see figure 6) in which the 6 curves (from left to right) represent the increasing polar angle and the 13 points on each curve represent the ratio 
between the two fluences (homogeneous / 24 points) calculated for that particular azimuthal angle. The study was repeated for different distances from the head surface.

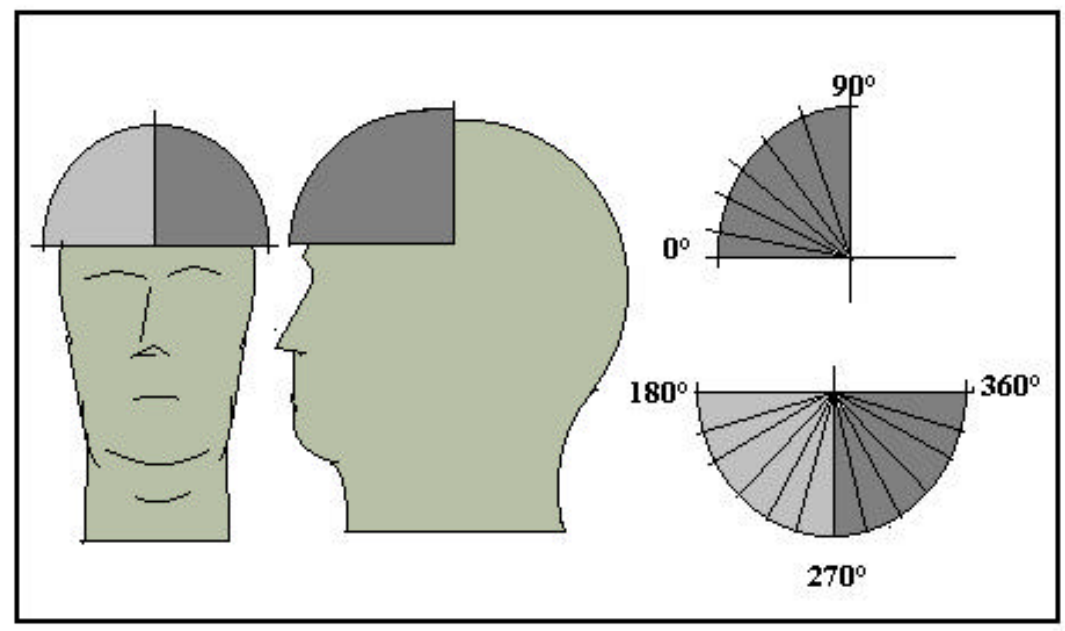

Fig.5 Sketch of the response spherical segment on the top of the head

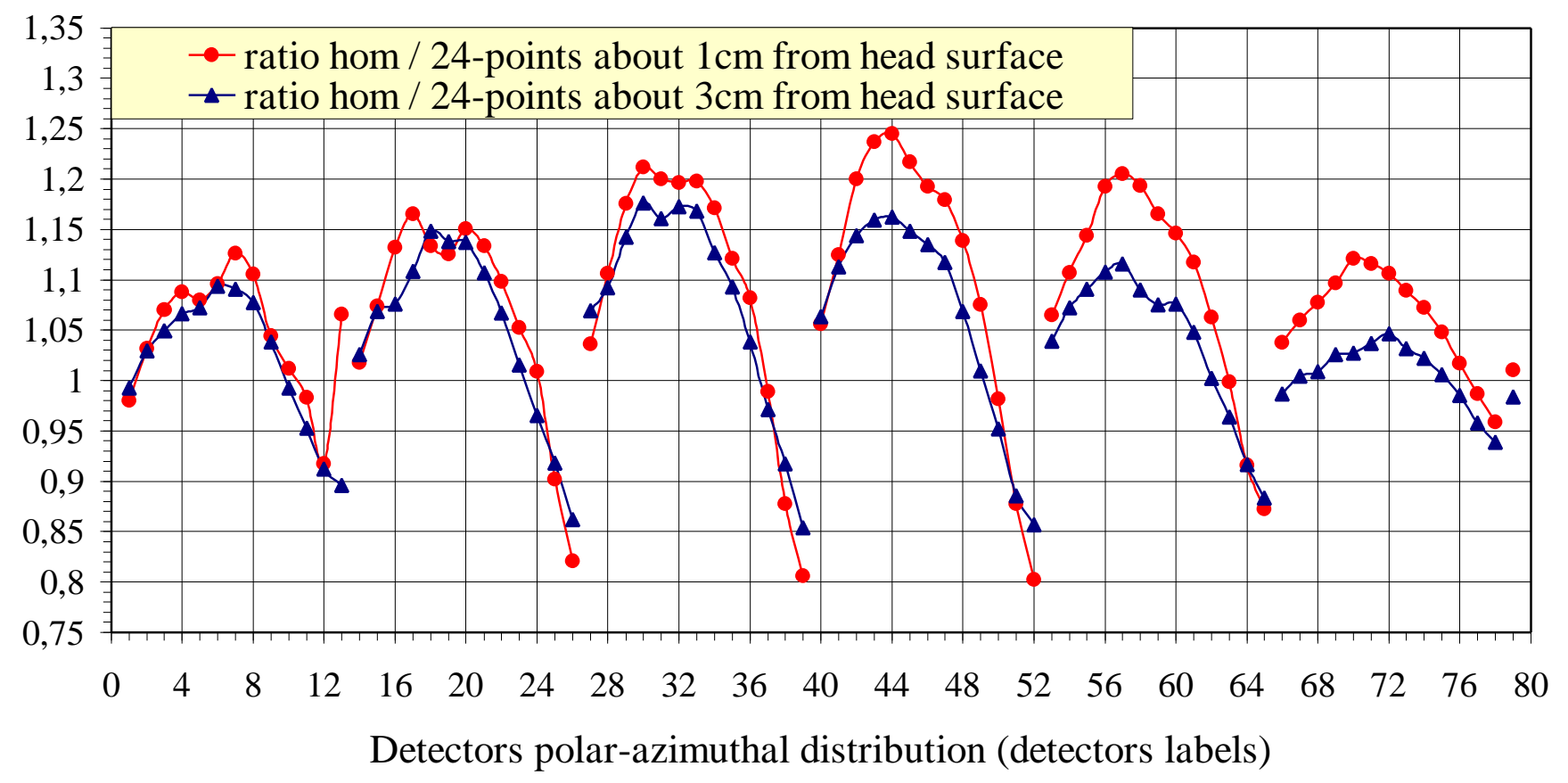

Fig.6: Behaviour of the fluence ratios in the 79 detection positions. 
Some conclusions can be drawn from these Monte Carlo calculations:

- The agreement between the two models (fully homogeneous and 24 sources heterogeneous) is bounded within maximum $\pm 20 \%$

- The agreement is within $\pm 10 \%$ for higher polar angles.

- The agreement is improved, as expected, at higher distances from the skull surface

\section{4-CALIBRATION MEASUREMENTS}

The measurement tests on the head phantom were carried out using the Whole Body Counter (WBC) operating at the ENEA Institute for Radiation Protection, in the Casaccia research centre. A detection system for low energy photons, equipped with 2 Canberra $^{\mathrm{TM}}$ LEGe detectors (each one with active area of $38 \mathrm{~cm}^{2}$ ), was used. The shieldeding is made (from external to internal) of $50 \mathrm{~cm}$ concrete, $20 \mathrm{~cm}$ iron, $3 \mathrm{~mm}$ lead, $2 \mathrm{~mm}$ copper and $1 \mathrm{~mm}$ cadmium. This system is frequently used for "in vivo" monitoring of actinides.

- The detector position, with respect to the head phantom, was chosen as a compromise between the need of minimising the photon fluence variation due to the heterogeneous source distribution and that of reaching a Minimum Detectable Activity (MDA) ${ }^{[7]}$ of the order of $3-4 \mathrm{~Bq}$ in the skull, in a reasonable measuring time (20-30 minutes).

\begin{tabular}{|c|c|c|c|}
\hline $\begin{array}{l}\text { Head surface to } \\
\text { detector distance } \\
\text { (cm) }\end{array}$ & $\begin{array}{c}\text { MCNP } \\
\text { (homogeneous } \\
\text { source) } \\
\text { Cps/Bq }\end{array}$ & $\begin{array}{c}\text { MCNP } \\
\text { (24 points heterogeneous } \\
\text { source) } \\
\text { Cps } / \mathbf{B q}\end{array}$ & $\begin{array}{c}\text { WBC } \\
\text { Cps/Bq }\end{array}$ \\
\hline$\sim 1$ & $(6.70 \pm 0.01) 10^{-3}$ & $(7.54 \pm 0.01) 10^{-3}$ & $(8.7 \pm 0.1) 10^{-3}$ \\
\hline$\sim \mathbf{1 0}$ & $(2.10 \pm 0.01) 10^{-3}$ & $(2.21 \pm 0.01) 10^{-3}$ & $(2.48 \pm 0.03) 10^{-3}$ \\
\hline$\sim 15$ & $(1.37 \pm 0.01) 10^{-3}$ & $(1.40 \pm 0.01) 10^{-3}$ & $(1.41 \pm 0.02) 10^{-3}$ \\
\hline$\sim 20$ & $(0.953 \pm 0.003) 10^{-3}$ & $(0.969 \pm 0.003) 10^{-3}$ & $(0.95 \pm 0.01) 10^{-3}$ \\
\hline
\end{tabular}




\begin{tabular}{|c|l|l|l|}
\hline $\begin{array}{c}\text { Head surface to } \\
\text { detector distance } \\
(\mathbf{c m})\end{array}$ & $\begin{array}{c}\text { MCNP } \\
\text { homogeneous -WBC } \\
\text { measur. } \\
\text { ratio }\end{array}$ & $\begin{array}{c}\text { MCNP } \\
\text { heterogeneous -WBC } \\
\text { measur. } \\
\text { ratio }\end{array}$ & $\begin{array}{c}\text { MCNP } \\
\text { Homogeneous-heterogeneous } \\
\text { correction factor }\end{array}$ \\
\hline$\sim \mathbf{1}$ & 0.77 & 0.87 & 0.89 \\
\hline$\sim \mathbf{1 0}$ & 0.85 & 0.89 & 0.96 \\
\hline$\sim \mathbf{1 5}$ & 0.98 & 0.99 & 0.99 \\
\hline$\sim \mathbf{2 0}$ & 1.00 & 1.02 & 0.98 \\
\hline
\end{tabular}

Table1 and Table 2: MCNP and experimental results for some detector configurations

Analysing the results achieved through the fluence mapping, it was decided to work in the neighbourhood of $60^{\circ}$ and $75^{\circ}$ polar angles.

Three different evaluations of full energy peak efficiency for the $59.5 \mathrm{keV}{ }^{241} \mathrm{Am}$ photon emission, were performed at different distances of the detector from the phantom surface by:

- Monte Carlo calculation simulating the phantom experimental conditions;

- Monte Carlo calculation simulating the real man contamination;

- experimental WBC measurements on the phantom.

A pulse height estimator was used in MCNP to evaluate the pulse number distribution as a function of the photon energy. The particles were scored only within the detector ROI, killing the photons below its lower limit, to reduce the computing time per tracked particle. The results are reported in tables 1 and 2 .

As expected the agreement among efficiency values obtained by the two Monte Carlo simulations (24 points-source, and homogeneous source) and the WBC measurements, progressively improves with the increase of the measurement distance, reaching a nearly perfect agreement at $15-20 \mathrm{~cm}$.

Taking into account that the required values of MDA (3-4 $\mathrm{Bq}$ of ${ }^{241} \mathrm{Am}$ in the skull) have been reached only with a 30 minutes measurement and with a distance of $1 \mathrm{~cm}$ between detector and 
head surface, the analysis of the results suggests the adoption of the following calibration procedure:

1- Perform the calibration measurements at $15 \mathrm{~cm}$ from the head surface of the phantom, in order to guarantee the accuracy, reducing the non-uniformity effects of 24 points distribution of sources.

2- Perform the "in vivo" measurement at $1 \mathrm{~cm}$ from the skull surface in order to reduce the counting time increasing detection efficiency.

3- Apply the proper correction coefficient CF for the calibration factor given by:

$$
\mathrm{CF}=\mathrm{CF}_{\mathrm{D}} \times \mathrm{CF}_{\mathrm{H}}
$$

where:

$\mathbf{C F}_{\mathbf{d}}=$ correction factor for different head to detector distance, for the homogeneous source, obtained from two MCNP calculation at measurement $(1 \mathrm{~cm})$ and at calibration $(15 \mathrm{~cm})$ distance, for the selected detection position.

$\mathbf{C F}_{\mathbf{H}}=$ correction factor for source heterogeneity, calculated comparing MCNP results, at calibration distance of $15 \mathrm{~cm}$, for the homogeneous and heterogeneous source distribution, for the same detection position.

In the adopted measurement configuration $(15 \mathrm{~cm}$ distance $), \mathbf{C F}_{\mathbf{d}}=\mathbf{4 . 8 9} \pm \mathbf{0 . 1}$ and $\mathbf{C F}_{\mathbf{H}}=\mathbf{0 . 9 9}$ $\mathbf{\pm 0 . 0 2}$ (Monte Carlo two standard errors) and therefore $\mathbf{C F}=\mathbf{4 . 8} \pm \mathbf{0 . 5}$ (95\% confidence interval including both the Monte Carlo precision and the MCNP complex head model accuracy that could be quantified within $\pm 10 \%$ ).

\section{CONCLUSIONS}

The procedure here presented was used to develop a calibration phantom in which a heterogeneous distribution of ${ }^{241} \mathrm{Am}$ sources well approximates a homogeneous distribution of the nuclide in the bone. The Monte Carlo code MCNP was used to quantify the level of approximation introduced and to evaluate correction factors for detection position and source heterogeneity. 
The comparison with the experimental measurements confirmed the reliability of the procedure, that could be adopted as a standard for the design of head calibration phantoms. Possible refinements of the methodology could be based on a larger number of point sources to be embedded in the head plastic matrix and a Monte Carlo model of the head based on a VOXEL phantom directly obtained from the CT scan Hounsfield raw data.

Acknowledgement: the authors are grateful to Dr.L.Pierotti (Health Physics Service, S.Orsola Hospital, Bologna) and Dr. G.Turci (Radiology Service "Canini”, S.Orsola Hospital, Bologna) for the CT scan analyses.

\section{REFERENCES}

[1] "Current status of the whole body counting as means to detect and quantify previous exposure to radioactive materials" Health $\mathrm{Ph}$. Vol 60. Supp. 1, pp 7-42, 1991.

[2] "Phantoms and Computational Models in Therapy, Diagnosis and Protection", ICRU report 48, 1992.

[3] "The Metabolism of Plutonium and Related Elements", Annals of ICRP publication 48, Vol. 16 o. 2/3 Pergamon Press. 1986.

[4] Alderson $^{\mathrm{TM}}$ Private Communication.

[5] J. F. Bresmeister (editor) “MCNP - A general Monte Carlo N-Particle Transport Code” Version 4B. “ LA-12625 Los Alamos National Laboratory (March 1997).

[6] Van Riper K. Sabrina User's Guide. White Rock Science, 1997.

[7] Currie L.A. “ Limits for qualitative detection and quantitative determination: application to radiochemistry" Analytical Chemistry, Vol.40 , N.3, March 1998. 